Biology | Janin Riedelsberger

\title{
Experimental and computational studies of plant ion channels
}

The yields and nutritional profiles of crops are affected by their intake of nutrients.
To understand how plant ion channels uptake and regulate sodium and potassium, Dr Janin Riedelsberger at the Center for Modeling (CBSM), University of Talca and her collaborators experimental approaches. The computational methods are used to model the channel protein's molecular structure and simulate its molecular dynamics, while experiments allow validation of the computational predictions. Conversely, computational data interpret experimental data. Bioinformatics, Simulation and

\section{Dermeable and separate the cell from the external environment. They act as barriers for various molecules. Transport proteins represent ways for molecules to pass across the membrane and regulate which ones gain access into the plant cell. The crucial role of membrane transport processes makes those described here, aimed at improving the nutritional value of plants and more. \\ To grow and develop, plants need various macronutrients including nitrogen phosphorous and potassium $\left(\mathrm{K}^{+}\right)$which they take up, along with sodium $\left(\mathrm{Na}^{+}\right)$ mostly from the soil via their roots. Specialised proteins (e.g., ion channels or}

ion. When the external $\mathrm{K}^{+}$concentration is higher, passive transport proteins, like ion channels, enable $\mathrm{K}^{+}$uptake.

Sodium is not essential for plants, but in small quantities it aids in metabolism and synthesis of chlorophyll. In some plants, it can partially replace potassium since sodium ions are chemically and physically similar to potassium ions. Due Pastions can cause symptoms of potassium deficiency in plants In themselves, high salt concentrations are a problem for most plants.

Plants respond in two phases to salinity: osmotic and ion-specific. In the osmotic phase (minutes to days), changes in
solute concentration (e. high salt)

Studies of how ion uptake occurs will help to explain why some plants are more tolerant to salinity stress than others.

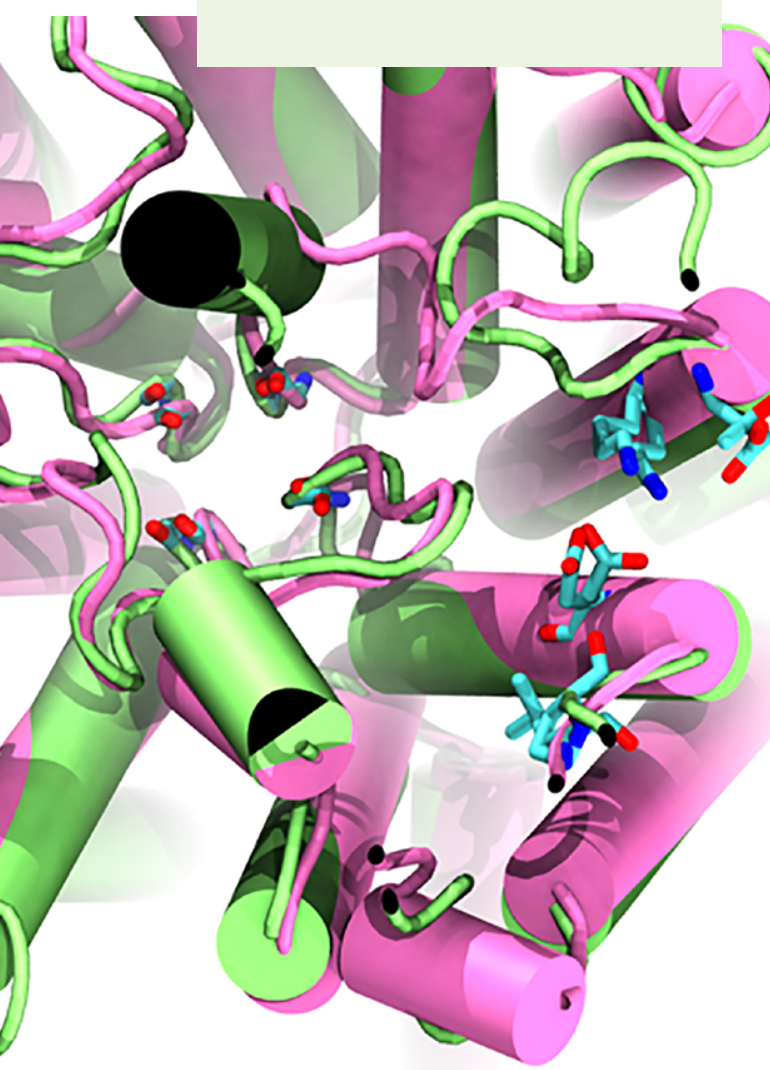
these ions under specific conditions so that they can be used by the plant in a
regulated way that ideally avoids toxicity.

Potassium, for example, is relevan in resistance to drought, salinity and fungal diseases, which is why it affects involved in the regulation of enzyme activities, in adjusting the electrical membrane potential and the cellular turgor, in regulating cellular homeostasis
and in stabilising protein synthesis.

Plants use different $\mathrm{K}^{+}$uptake systems, depending on the externally available $K^{\prime}$ concentration, to keep the cellular concentration of this constantly high. If the external $\mathrm{K}^{+}$ concentration is very low, active uptake systems are used, which often co- cause changes in water movement and consequently growth reduction. In the start accumulating in toxic concentrations, metabolic processes are affected and cell death occurs if no measures are taken.

Studies of how ion uptake occurs will help to explain, for example, why some plants are more tolerant to salinity stress than others, and could enable breeders to develop crop plants that are more cesistant. Such work is being advanced by molecular structures of the ion channels involved, and then guide experiments or explain experimental observations.

The Center for Bioinformatics, Simulation and Modeling (CBSM) combines proteins with molecular dynamics mulations to study protein-membran systems over time and in response to recently, a transport protein of the HKT family (OsHKT22) in rice (Onza sativa) was studied by computational and experimental methods.

\section{THE HKT PROTEIN FAMILY}

The family of HKT proteins was initially named for 'high-affinity potassium' but was then found to be also involved in sodium transport. There are two kinds of HKT channels: class I are mainly $\mathrm{Na}$ selective (called uniport), while class II ac as $\mathrm{Na}^{+}$and $\mathrm{K}^{+}$cotransporters (sympor). The second type has been dry-lab (meaning computer) modelled and

Comparative modelling of this HKT

membrane protein followed by simulation of the protein dynamics performed by the stes involved in ion conduction across the membrane. One of the coordination sites was then validated experimentally using electrophysiological methods.

The family of HKT proteins is functionally diverse in ion selectivity and the effect that the external cation composition have on the protein's function.

Monocotyledons like rice appear to have a higher variety of HKT proteins than do dicotyledons, and it is only within these kinds of $H K T$ channels that cation sympor is found. OsHKT2,2 is one of eight or nine cultivar) found in rice.

The ion composition in the extracellular ransport abilities of HKT channels by changing their ion transport rates. Dr Janin Riedelsberger and her colleagues set out to study this transportation

process at the molecular level, successfully creating a molecular homology model of rice OsHKT2;2.

Computational approaches like comparative modelling are used to show what the structure of the protein HKT could look like, and to predict how the structure of a protein affects its function. Then, he researchers have to design wet-lab experments (for example, their predictions.
Cation coordination site on the
extracellular protein surface of
OSHKT T2:2 Negatively charge charged $\mathrm{Na}^{+}$ion (yellow). The positively charged nitrogen atom
(blue) of Iysine is crucial for the

formation and fun
coordination site.
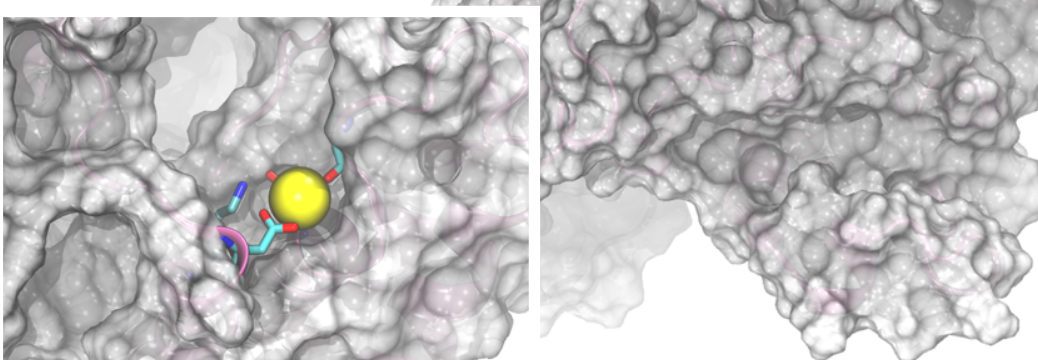

Dy-lab experiments show where io approach the protein but not how they The next step is to mutate these poten. The next step is to mintate these potential in the protein structure) and see whether these changes affect the function of $\mathrm{HK}$. If they do, this shows that a site is important for the function. If there is no change, this part of the protein is not crucial for protein function.

COMPARATIVE PROTEIN MODELLING Comparative protein modelling involves constructing a model of a protein from tis amino acid sequence based on the thre dimensional structure of a homologous protein (a protein that resembles the protein of interest). Bacterial and fungal Ktr and Tik channels, which have been extensively studied, are homologues

The structural model of OsHKT2;2 was computer-generated using the

for automated protein structure and unction prediction. Starting from an amino did sequence, - Tasser generates threedin A comparative model was created for the class II-type HKT channel OSHKT2:2 and mbedded in a lipid membrane.

The team then simulated the molecule's dynamics in the presence of $\mathrm{Na}^{+}$and $\mathrm{K}^{-}$ ions and evaluated where ions approached and interacted with the channel protein. Several cation coordination sites were extracellar surface of the be pros protein where ions must pass to reach the inside of the cell.

HKT proteins have a structure composed of one amino acid chain that contains segments and one pore lop. These for units group together to form a conduction pathway (pore) in the center of the protein. In the class II type of HKT studied each of

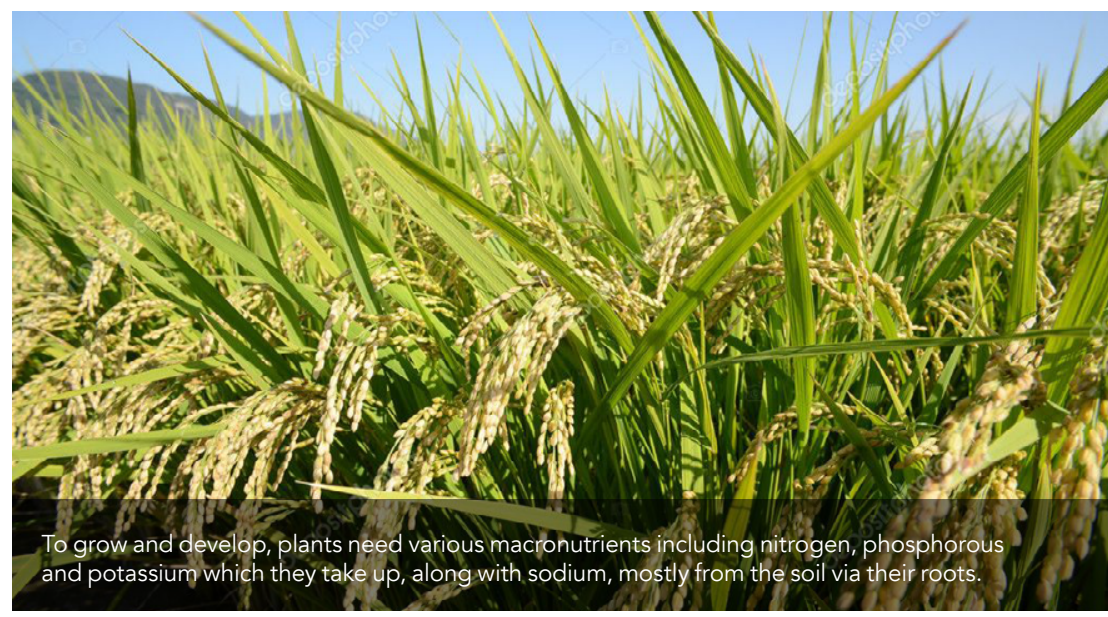


Electrophysiological characteristics of OsHKT2;2 in X. laevis oocytes

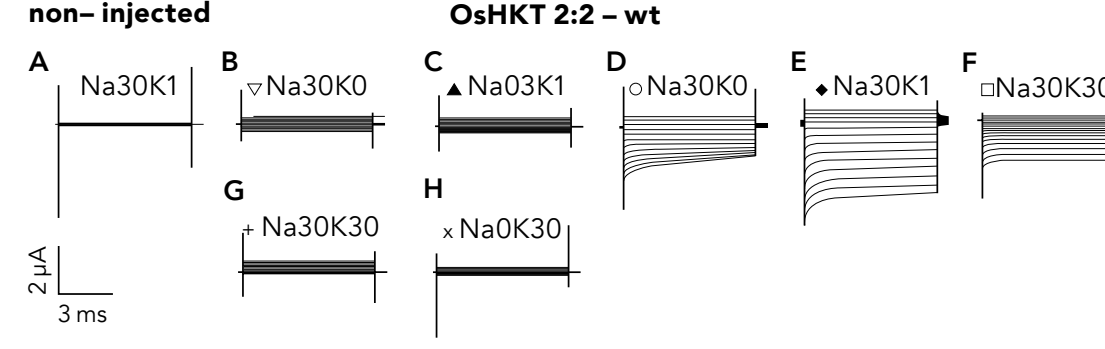

A: Representative currents elicited in control cells (not injected with cRNA). B-H: Representative of variable ion concentrations - $\mathrm{Na}$ 0/03/30: 0/0,3/30 mM NaCl, KO/1/30: 0/1/30 mM KCl. From Riedelsberger et al. BMC Plant Biology 2019, under the terms of the
International License (http://creativecommons.org/licenses (by/4.01).

the units contained a conserved glycine in The current through ion channels is its pore loop (G-G-G-G), while in class I the affected by dynamic changes in the first glycine is mutated to serine (S-G-G-G). channel conformation during gating

ELECTROPHYSIOLOGICAL (the process by which the channel or pore

\section{MEASUREMENTS} in a cell membrane opens or closes).

lectrophysiology is an example of a To identify the amino acids that are study the flow of ions through an actual pore, the researchers constructed a mode channel in biological tissues. As mentioned of the wild-type channel protein based

Computational approaches provide insights into structure-function relations linked to ion conduction in complex proteins like HKT channels.

earlier, wet-lab experiments are designed on structurally- and functionally-related to help verify whether the predictions bacterial channels. They described three made by computational approaches (d lab experiments) are correct. functional (electrophysiological) properties for wild-type OstKy 2,2 and ex pained the occurence and characteristics of these
properties in mutant channels. In this case, a voltage clamp technique was used to characteise the

OSIKT2 2 plogical properties of rice In other words, to study what role the coordination site plays in the

expressed in frog eggs (Xenopus laevis.

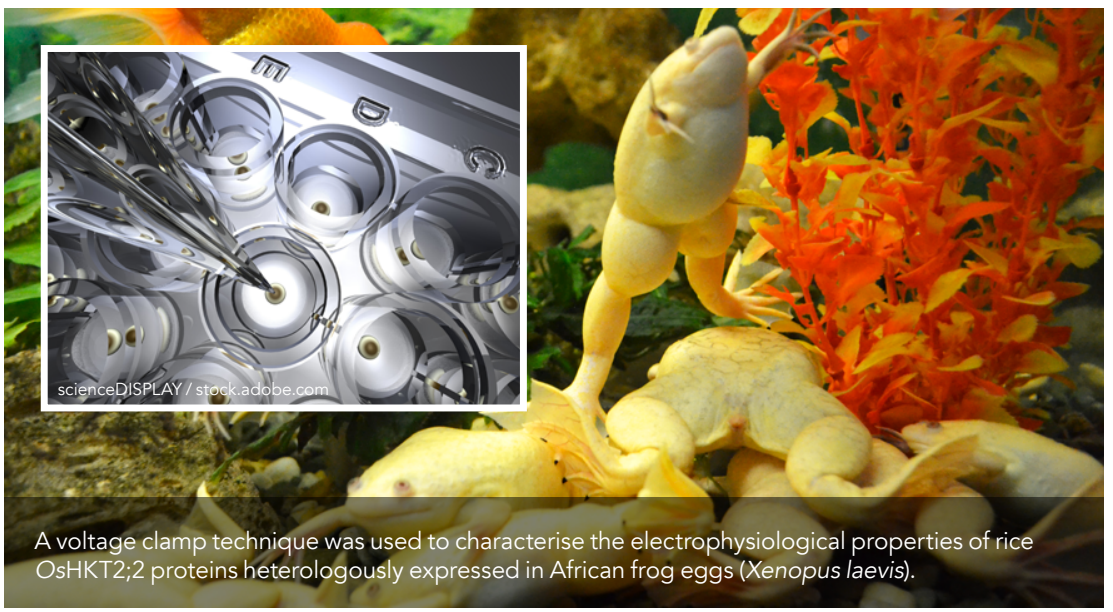

residues (specific amino acids which form mutated and then the protein) were experimentally charectersed for changes in functionality One mutant (K504) had significant effect on the channel's function compared to the wild-type channel.

\section{DENTIFICATION OF A CATION} COORDINATION SITE

Analyses of the contact frequency between ions and amino acids during molecular dynamics simulations enabled identification of a potential extracellular cation coordination site located in the outer extracellular protein region some distance $(20 \mathrm{~A})$ from the pore entrance of OsHKT 2,2. Coordination sites like the

The computational studies found that the extracellular cation coordination site consisted of a positively charged lysine charged aspartates in place to form a negatively charged environment th attracts cations. Side chain oxygen atoms of the two aspartates, together with the backbone oxygen of a third residue, appeared to capture $\mathrm{Na}$ and $\mathrm{K}^{+}$ions from the extracellular space and put them on a path that guides ions towards the pore and into the cell.

From this, the researchers suggested that the positively charged lysine could be the impulse that cations need to be moved towards the pore. The positive charge and its correct size and posit functionality. The sensitivity towards changes of one of the parameters in size or charge indicates how finely tuned the structure of the coordination site in the plant's transporter protein is.

The coordination site was found to be essential for proper ion conduction. Crucial sites, like this, on the extracellular protein surface could help show how different ion species may affect channe conduction behaviour even before entering the pore. Research at CBSM s contributing to the understanding of how $\mathrm{Na}^{+}$and/or $\mathrm{K}^{+}$transporting proteins work, which help to explain how plants react under salt stress situations and how they cope with increased
ions in the plant cell for example.
Research Objectives

Dr Riedelsberger's primary research interest explores De relationship between the steucture and function

\section{Detail}

Janin Riedelsberger

Center for Bioinformatics, Simulation and Modeling (CBSM), Engineering Faculty, University of Talca, 2 Norte 685 , 3460000 Talca, Chile

Bio

Dr Riedelsberger studied Biology at University of Potsdam, Germany (2003-2009). She completed her PhD at the University of Potsdam and MaxPlanck-Institute for Molecular Plant Physiology financed by a fellowship of the International Max Planck Research School (2009-2013). Following that she moved to Chile in 2014 and began her Postdoc fellowship. In 2017, Dr Riedelsberger started an academic position at the University of Talca financed by the project PAl from Conicy.

Funding

(Científico y Tecnológico (Conicyt), programmes Fondecyt and PAI

Collaborators

- DrWendy Gonzaléz

- Dr Miguel A. Piñeros

- Dr Ariela Vergara-Jaque

\section{TCDCA 2 CBSM}

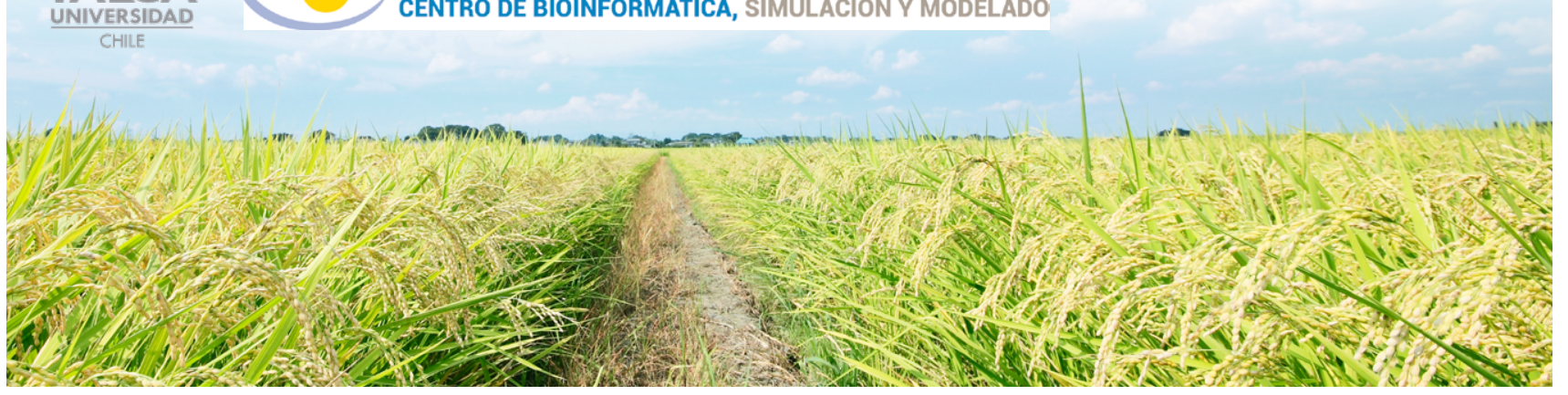

\section{References}

Riedelsberger, J., Vergara-Jaque, A., Piñeros, M., Dreyer, I. \& Gonzalez, W. (2019). An extracellular cation coordination site influences ion conduction of
OsHKT2:2. BMC Plant Biology 19, 316. doi:10.1186 s12870-019-1909-5

Sklodowski, K., Riedelsberger, J., et al. (2017). The voltage-gated potassium chann

Sharma, T., M., Dreyer, Riedelsberger, J., et al. (2013). of potassium in the model plant Arabidopsis thalian fpls.2013.00224

\section{Personal Response}

Please tell us more about your future plans for this work in developing and testing promising rice cultivars experimentally?

III CBSM's main focus is the structure-function investigation of a variety of proteins involved in diverse cellular processes. The aim is to explore and characterise protein structures to understand their function, which is tightly connected to the structure. been identified, it is tested using the actual in a biological environment. receptor-like pseudokinase MRH1 interacts with the
voltage-gated potassium channel AKT2. Sci Rep. 7: The role of $K^{+}$channels in uptake and redistribution 